\title{
Ações para divulgação da Neurociência: um relato de experiências vivenciadas no sul do Brasil
}

\author{
Actions for Neuroscience propagation: a report of experiences in southern Brazil
}

\author{
Alexandre Martins ${ }^{1 *}$, Pâmela Billig Mello-Carpes ${ }^{2^{*}}$ \\ ${ }^{1}$ Acadêmico do Curso de Fisioterapia na Universidade Federal do Pampa, UNIPAMPA, Campus Uruguaiana, \\ bolsista do Grupo de Pesquisa em Fisiologia, Uruguaiana, RS, Brasil. \\ 2 Professora Adjunta na UNIPAMPA, Campus Uruguaiana, líder do Grupo de Pesquisa em Fisiologia. \\ *e-mail: pamelacarpes@unipampa.edu.br
}

Agências de fomento: Programa Novos Talentos - Coordenação de Aperfeiçoamento de Pessoal de Nível Superior CAPES/Brasil; Sociedade Brasileira de Fisiologia; Sociedade Brasileira de Neurociências e Comportamento, Pró-reitoria de Extensão da Universidade Federal do Pampa

\section{Resumo}

Recentemente a popularização e divulgação da ciência têm ganhado significativa expansão. As ciências relacionadas ao cérebro, tais como a neuroquímica e a neurofisiologia se destacam neste cenário. Uma iniciativa que têm auxiliado muito neste processo é a Semana Internacional do Cérebro, que contribui para a divulgação dos benefícios da investigação científica nesta área. Relatamos ações de divulgação e popularização da neurociência, realizadas na cidade de Uruguaiana/RS/Brasil durante a Semana Internacional do Cérebro em 2013. Foram realizadas ações variadas em diferentes contexto e ambientes da cidade. Os resultados desta proposta mostram a importância de ações para divulgação da neurociência e neuroquímica junto à população. As ações atenderam ao objetivo de popularizar conceitos científicos e foram muito bem aceitas pelo público participante, permitindo a aproximação da comunidade com a universidade e com os discursos e práticas da neurociência e suas áreas.

Palavras-chave: Divulgação da ciência; Popularização da ciência; Cérebro.

\begin{abstract}
Recently the popularization and dissemination of science have gained significant expansion. The sciences related to the brain such as neurochemistry and neurophysiology stand out in this scenario. An initiative that would have helped a lot in this process is the Brain Awareness Week, which contributes to the dissemination of the benefits of scientific research in this area. Here we report actions of dissemination and popularization of neuroscience held in the city of Uruguayana/RS/Brazil during the Brain Awareness Week in 2013. Various actions in different contexts and environments were conducted. The results show the importance of the proposed actions for dissemination of neuroscience and neurochemistry to the population. Actions meeting the first objective of popularizing scientific concepts and were very well accepted by participating public, allowing the community approach with the university and with the discourses and practices of neuroscience and its areas.
\end{abstract}

Keywords: Disclosure of science; Popularization of science; Brain. 
Imagem Pública E Divulgação Científica Da Bioquímica: Ações para divulgação da Neurociência: um relato de experiências vivenciadas no sul do Brasil

\section{Introdução}

Recentemente a produção de novos conhecimentos na área da Neurociência tem tido um intenso crescimento. A década de 90, por exemplo, ficou conhecida como a "Década do Cérebro", em virtude da ênfase na investigação do Sistema Nervoso Central (SNC), seja do ponto de vista psicológico, genético, fisiológico, biofísico, bioquímico, ou tantos outros que compõem as pesquisas em Neurociência [1].

A neurociência constitui um conjunto das diversas áreas do conhecimento que pesquisam o Sistema Nervoso (SN). Estas áreas estudam as moléculas que constituem os neurônios, os órgãos do SN e suas funções específicas, bem como o comportamento humano resultante da atividade dessas estruturas. Podemos dividir a neurociência em subáreas, tais como neuroanatomia, neurofisiologia, neuroquímica... incluindo uma área mais recente, a nova área da neuroeducação. Esta última surge porque se percebeu que os avanços da neurociência esclareceram muitos aspectos acerca do funcionamento do SNC, especialmente do cérebro, e permitiram a abordagem mais científica do processo ensino-aprendizagem.

Sabendo da importância do SN enquanto um sistema de controle que influencia a homeostasia corporal e os demais sistemas orgânicos de nosso corpo, torna-se importante divulgar e popularizar a neurociência e suas áreas afins, permitindo que a população se aproprie de saberes que garantirão uma maior autonomia e, ainda, permitindo que adote atitudes condizentes com a manutenção da saúde do cérebro e SN. A popularização da ciência como um todo assume um caráter fundamental porque permite uma proximidade das pessoas com o discurso da ciência, desvendando o seu caráter histórico e humano, a sua proximidade com o senso comum, sem o qual perderia todo o sentido [2]. Para Candotti [3], "a popularização do que desconhecemos é tão ou mais importante do que a popularização do que conhecemos".

Ainda, é extremamente importante que as descobertas científicas sejam divulgadas de forma adequada à população. Candotti [4] acredita que "a circulação das ideias e dos resultados de pesquisas é fundamental para avaliar o seu impacto social e cultural, como também para recuperar, por meio do livre debate e confronto de ideias, os vínculos e valores culturais que a descoberta do novo, muitas vezes rompe ou fere". Na Conferência das Nações Unidas sobre Meio Ambiente e Desenvolvimento de $1997^{1}$ definiu-se no

\footnotetext{
${ }^{1}$.http://www.redemulheres.gov.br/responsabilidade-socioambiental/agenda-21/agenda-21-

brasileira/itemlist/category/107-agenda-21? start $=60$
} 
Imagem Pública E Divulgação Científica Da Bioquímica: Ações para divulgação da Neurociência: um relato de experiências vivenciadas no sul do Brasil

Capítulo 31.1 que "deve-se ajudar o público a comunicar à comunidade científica e tecnológica suas opiniões sobre como a ciência e a tecnologia podem ser melhores gerenciadas para influir beneficamente na vida dele".

Com o intuito de auxiliar a divulgação da ciência relacionada ao cérebro foi criada a Semana Internacional do Cérebro (SIC). A SIC é uma campanha global que visa promover a conscientização do público em geral sobre os avanços e benefícios da pesquisa e divulgação da neurociência. Esta ação acontece sempre em março, quando vários parceiros em todo o mundo celebram a semana do cérebro através de diversas atividades de divulgação científica voltadas para pessoas de todas as idades. A iniciativa de realizar a SIC em Uruguaiana (Rio Grande do Sul, Brasil) nasceu quando os membros do Grupo de Pesquisa em Fisiologia (GPFis) da Universidade Federal do Pampa (Unipampa) tomaram conhecimento deste evento, cuja organização é liderada pela Dana Alliance for Brain (DANA) $)^{2}$ e este artigo tem como objetivo relatar as ações realizadas na SIC em Uruguaiana.

\section{Materiais e Métodos}

A primeira edição da SIC em Uruguaiana/RS foi realizada entre 11 e 17 de março de 2013, sendo organizada por membros do GPFis/Unipampa

Todas as atividades propostas foram abertas à participação da comunidade acadêmica da UNIPAMPA e da população de Uruguaiana, incluindo alunos da rede pública de Educação Básica e a população em geral. As atividades aconteceram na Unipampa, na sede do Serviço Social do Comércio (SESC) de Uruguaiana e em um parque público municipal (Parque Dom Pedro II).

As atividades foram divulgadas através de cartazes distribuídos em locais estratégicos no centro da cidade, na universidade e nas escolas de Uruguaiana, de redes sociais e na página do GPFis. As escolas públicas de Educação Básica ainda receberam convites para participação em atividades específicas. As atividades realizadas estão detalhadas no Quadro 01.

${ }^{2}$ DANA: http://www.dana.org/; Brain Awareness Week/DANA: http://www.dana.org/BAW/ 
Imagem Pública E Divulgação Científica Da Bioquímica: Ações para divulgação da Neurociência: um relato de experiências vivenciadas no sul do Brasil

Quadro 1. Atividades realizadas durante a SIC 2013 de Uruguaiana/RS.

\begin{tabular}{|l|l|}
\hline Atividade & Descrição \\
\hline 1) Oficina de neuroanatomia & $\begin{array}{l}\text { Esta oficina prática procurou abordar os aspectos mais específicos da } \\
\text { anatomia do SN utilizando peças anatômicas. Foi realizada no Laboratório } \\
\text { da Anatomia Humana do campus Uruguaiana da Unipampa. Público-alvo } \\
\text { principal: estudantes de graduação ingressantes. }\end{array}$ \\
\hline $\begin{array}{l}\text { 2) Oficina de neurofisiologia e } \\
\text { neuroquímica da } \\
\text { aprendizagem e da memória }\end{array}$ & $\begin{array}{l}\text { Esta oficina procurou discutir a neurofisiologia e a neuroquímica, } \\
\text { relacionando-as com as funções das diferentes regiões cerebrais, } \\
\text { facilitando, assim, a compreensão das funções de aprendizagem e } \\
\text { memória. Público-alvo principal: estudantes de graduação. }\end{array}$ \\
\hline $\begin{array}{l}\text { 3) Mostra de neuroanatomia funções foi } \\
\text { e neurofisiologia }\end{array}$ & $\begin{array}{l}\text { A fim de divulgar conhecimentos a respeito do SN e suas fun } \\
\text { realizada uma atividade com exposição de peças anatômicas, bem como } \\
\text { palestras explicativas e demonstrações práticas no centro da cidade. } \\
\text { Público-alvo principal: Estudantes da rede pública de Educação Básica. }\end{array}$ \\
\hline 4) Palestras diversas & $\begin{array}{l}\text { Palestras diversas foram realizadas com temáticas variadas: atenção e } \\
\text { tempo de reação; linguagem; e, exercício físico e memória. Público-alvo } \\
\text { principal: Público em geral. }\end{array}$ \\
\hline $\begin{array}{l}\text { 5) Atividade "Levando o } \\
\text { cérebro ao parque" }\end{array}$ & $\begin{array}{l}\text { Para divulgar conhecimentos gerais sobre o SN e sobre sua importância } \\
\text { foram realizadas ações no parque público municipal de Uruguaiana } \\
\text { (Parque Dom Pedro II), envolvendo distribuição de folders com } \\
\text { informações gerais sobre o cérebro e o SN, aplicação de testes cognitivos, } \\
\text { demonstrações práticas acerca do funcionamento do sistema sensorial } \\
\text { (quimiocepção, visão, audição, etc) e instruções sobre a manutenção da } \\
\text { saúde do SNC. Público-alvo principal: Público em geral. }\end{array}$ \\
\hline $\begin{array}{l}\text { 6) Atividade "Como as drogas } \\
\text { agem no seu cérebro?" }\end{array}$ & $\begin{array}{l}\text { Espaço aberto onde contamos com pôsteres e distribuição de folders } \\
\text { autoexplicativos sobre como algumas drogas agem em nosso cérebro. } \\
\text { Também foi utilizado um software interativo para demonstrar o mecanismo } \\
\text { de ação de diferentes drogas no cérebro. Público-alvo principal: Público } \\
\text { em geral. }\end{array}$ \\
\hline
\end{tabular}

Após a SIC a equipe organizadora se reuniu para discutir o resultado das ações. As impressões relatadas foram anotadas e utilizadas como base para a próxima sessão deste artigo.

\section{Resultados e discussão}

A ideia de organizar a SIC foi desafiadora para o grupo proponente, primeiramente, por se tratar da primeira edição, mas também porque tivemos que pensar em atividades que envolvessem um grande e diversificado público, considerando o objetivo da proposta. Neste sentido propomos atividades simples, mas que envolvessem muito componente prático (Figura 01), assim, pudemos verificar que os objetivos iniciais foram plenamente atingidos. 
Imagem Pública E Divulgação Científica Da Bioquímica: Ações para divulgação da Neurociência: um relato de experiências vivenciadas no sul do Brasil
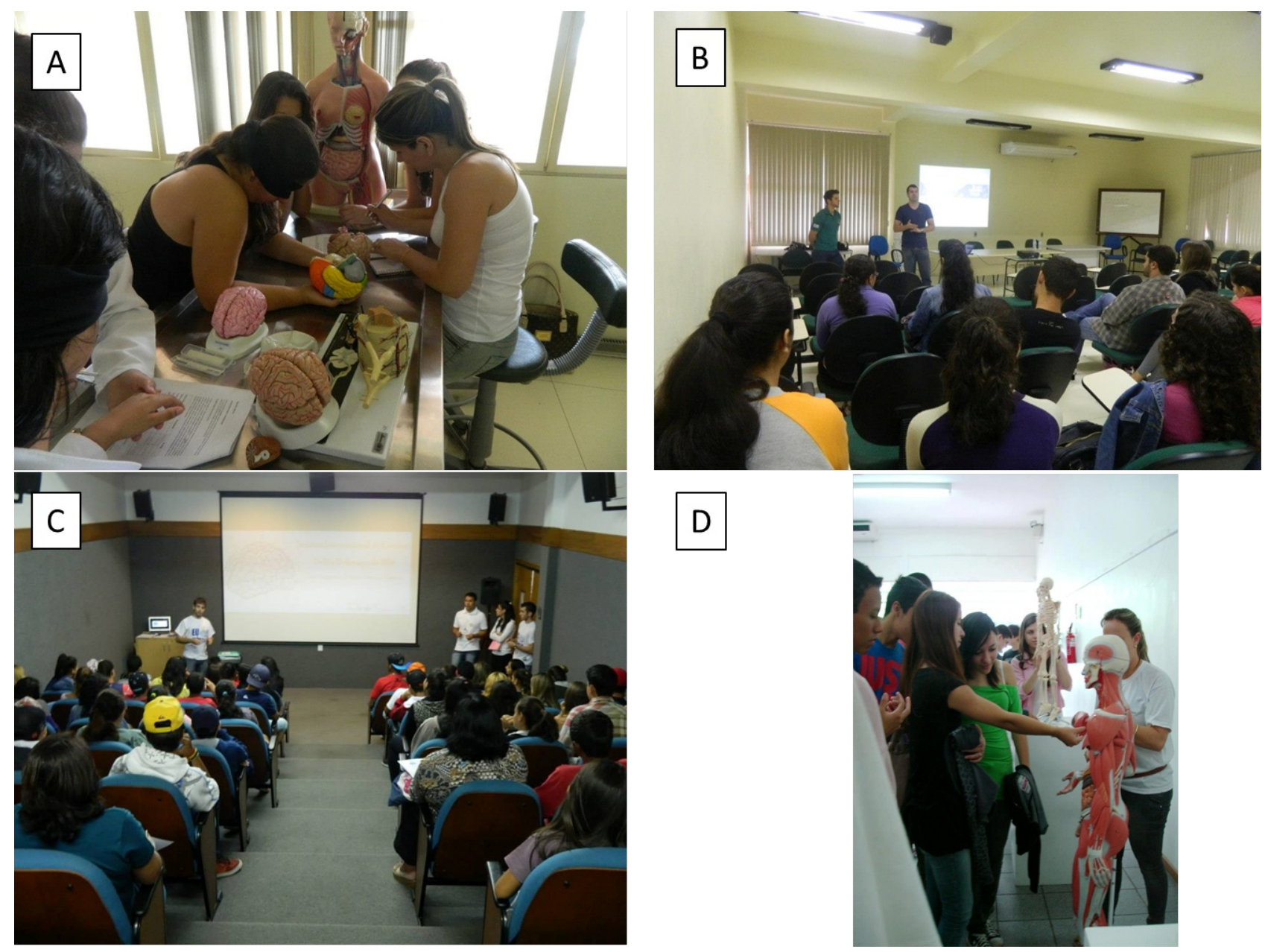

Figura 1. Imagens ilustrativas das atividades realizadas na SIC 2013 de Uruguaiana. 1 A e 1 B representam as oficinas e palestras; $1 \mathrm{C}$ e $1 \mathrm{D}$ a mostra de neuroanatomia e neurofisiologia.

Cerca de 1000 pessoas participaram de nossas ações. Não é possível estabelecer um número exato porque atividades como a "Mostra de Neuroanatomia e Neurofisiologia", "O cérebro vai ao parque" e "Como as drogas agem no seu cérebro?" receberam muitos visitantes, e nem todos assinaram o livro de participação (quadro 02). Foi impossível controlar esta variável, dado o grande número de visitas simultâneas que recebemos.

Quadro 2. Número de participantes por atividades d a SIC 2013 de Uruguaiana/RS.

\begin{tabular}{|l|l|}
\hline Atividade & Número de participantes \\
\hline 1) Oficina de neuroanatomia. & 30 \\
\hline $\begin{array}{l}\text { 2) Oficina de neurofisiologia e neuroquímica da aprendizagem e da } \\
\text { memória }\end{array}$ & 60 \\
\hline 3) Mostra de neuroanatomia e neurofisiologia & Aproximadamente 400 \\
\hline 4) Palestras diversas & 120 \\
\hline 5) Atividade "Levando o cérebro ao parque" & Aproximadamente 150 \\
\hline 6) Atividade "Como as drogas agem no seu cérebro?" & Aproximadamente 300 \\
\hline Público total & Aproximadamente 1060 \\
\hline
\end{tabular}


Imagem Pública E Divulgação Científica Da Bioquímica: Ações para divulgação da Neurociência: um relato de experiências vivenciadas no sul do Brasil

Além do grande público que participou da SIC 2013, muitos participantes relataram que as atividades foram positivas e acrescentaram novos conhecimentos. Um dos objetivos desta atividade foi transmitir novos conhecimentos através da divulgação cientifica. A divulgação científica vem se fixando no meio social com o intuito de estimular uma apropriação de conhecimentos e realizar a inclusão social como forma de promoção de cidadania [5]. Na divulgação de ciência em espaços não formais, como aqui proposto, a questão da neurociência e suas áreas também vêm ganhando destaque, principalmente com o objetivo de transformar o ser humano em um ator consciente no que tange processos envolvendo o cérebro. Para Guimarães e Vasconcelos [6], a educação não formal possui uma maior maleabilidade com relação ao tempo, e sua contribuição científica e humanística não tem a intenção de suprir deficiências do sistema formal, mas sim agir como um complemento que estimule a extensão do conhecimento através da interdisciplinaridade e contextualização.

É interessante destacar as atividades realizadas junto à Mostra de Neuroanatomia e Neurofisiologia, nas quais, além do público que foi visitar espontaneamente, recebemos a visita de turmas de estudantes de quatro escolas públicas da cidade. Os alunos da Educação Básica participaram ativamente de todas as atividades propostas e também obtivemos um retorno satisfatório por parte dos professores que os acompanhavam. Um aspecto importante a se considerar neste tipo de atividade é a necessidade de adaptar o conteúdo científico trabalhado, no nosso caso conceitos de neuroanatomia, neurofisiologia e neuroquímica, para uma linguagem acessível e de fácil compreensão para crianças e adolescentes. Cunha [7] escreve que para a ciência poder ser divulgada ao grande público é necessário realizar transformações no gênero discursivo e então a dita ciência acadêmica passa a ser uma ciência informativa. Zamboni [8] concorda, ressaltando que é preciso modificar a linguagem da ciência quando esta ultrapassa os muros da comunidade científica e chega aos olhos e ouvidos do homem comum.

Segundo Sarmento [9], quando se trata de divulgação científica para o público infantojuvenil, a linguagem precisa então ser ainda mais adaptada, com recursos metalinguísticos específicos, como vocabulário fácil, centrado no cotidiano, explicando e definindo termos científicos, com predominância do caráter conotativo, que recorre a comparações, analogias e metáforas, trazendo para o mundo do público e de vivência diária do público. Zamboni [8] destaca que quando a divulgação científica é destinada ao público adulto, a organização textual se dá sob a forma da argumentação, enquanto que 
Imagem Pública E Divulgação Científica Da Bioquímica: Ações para divulgação da Neurociência: um relato de experiências vivenciadas no sul do Brasil

quando é destinada ao público infantil ocorre ênfase na narrativa.

Outro aspecto relevante que destacamos em nosso encontro de avaliação das ações é a importância da preparação da equipe no que diz respeito ao conhecimento dos temas a serem trabalhados, além da criatividade na hora da elaboração das atividades. Segundo Carvalho [10] é importante entender o desenvolvimento do conteúdo a ser ensinado nos três aspectos (ciência, tecnologia e sociedade) que direcionam o ensino para uma finalidade cultural mais ampla, e esse ensino deve ser tal que leve os estudantes a construir o seu conteúdo conceitual e participar do processo de construção, tendo a oportunidade de aprender a argumentar e exercitar a razão, em vez de fornecerIhes respostas definitivas ou impor-Ihes seus próprios pontos de vista. Assim evitamos a transmissão de uma visão fechada de ciência.

Percebemos que a cada dia, em cada ação, éramos surpreendidos com novos e diferentes questionamentos que o público, especialmente o jovem, levantava durante as atividades. Nascimento Junior e Souza [11], em seu estudo, concluíram que na ação conjunta há troca vivências, socialização, questionamentos, reflexão, possibilidade de avaliação dos conhecimentos e capacidades dos educandos e mesmo uma prática mais crítica e dinâmica por parte do educador, pois nestas atividades é necessária uma intensa reflexão para que se possa melhor orientar o seu desenvolvimento.

A atividade que mais envolveu a população geral, incluindo pessoas de todas as idades, nem todos com algum nível de escolaridade, foi a proposta "Levando o cérebro ao parque" (figura 2). Muitas das pessoas que participaram não tinham nenhum conhecimento prévio de conceitos e da importância da neurociência. Esse momento foi de popularização e divulgação não só da neurociência, mas sim da ciência como todo. Teixeira [12] diz que a disseminação da informação tem significativo papel na construção do conhecimento e na formação da cidadania. São vários os argumentos do porquê de popularizar a ciência, os mais frequentes estão relacionados com: inclusão social, controle popular da ciência e tecnologia, erradicação de mitos, desenvolvimento econômico, dentre outros [2]. 
Imagem Pública E Divulgação Científica Da Bioquímica: Ações para divulgação da Neurociência: um relato de experiências vivenciadas no sul do Brasil
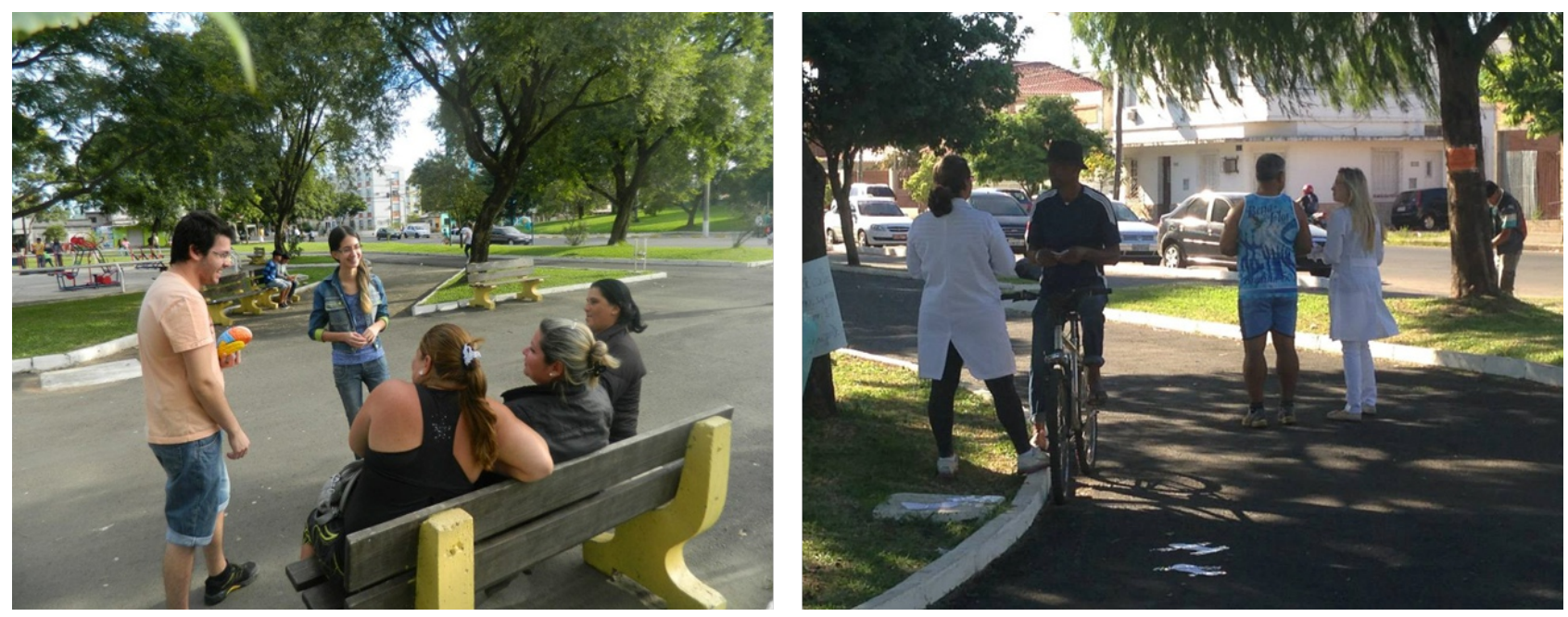

Figura 2. Imagens ilustrativas da atividade "Levando o cérebro ao parque".

Diante o exposto percebe-se a importância da divulgação da neurociência para a população, destacando-se os benéficos que ela traz através das pesquisas realizadas na área e tentando desmistificar a ideia que neurociência é coisa de cientista ou de laboratório. Adestro [13], em seu estudo com o uso da mídia televisiva, observou que a contribuição do potencial da abordagem transdisciplinar no processo de divulgação em neurociências promove uma informação que prima pela desmistificação de preconceitos e quebra de estigmas. Nós percebemos que nossa proposta também conseguiu promover esta desmistificação, além de promover uma aproximação entre a universidade e a comunidade, seja ela a comunidade escolar ou o público da cidade em geral.

\section{Impactos no ensino e na divulgação científica}

Durante as atividades realizadas na SIC muitas pessoas tiveram contato com nossas propostas, pessoas estas com idades variadas e diferentes níveis de escolaridade. É difícil quantificar o impacto que as atividades tiveram sobre a vida das dessas, mas temos convicção que trocamos conhecimentos, criamos discussões relacionadas às temáticas e provocamos o interesse do público sobre o tema e sobre a ciência de modo geral. Este é um passo importante, especialmente se considerarmos que a cidade de Uruguaiana/RS recebeu uma instituição pública de ensino superior há somente 7 anos, e grande parcela da população local desconhece o funcionamento da universidade, seus objetivos e até mesmo o fato de ela oferecer ensino superior totalmente gratuito. Assim, as ações desenvolvidas, além de aproximarem a comunidade de conceitos científicos, permitindo a visualização de suas aplicabilidades, aproximaram a 
Imagem Pública E Divulgação Científica Da Bioquímica: Ações para divulgação da Neurociência: um relato de experiências vivenciadas no sul do Brasil

universidade da sociedade, despertando o interesse por ela e seus fazeres.

Em relação do ensino o impacto das ações foi ainda mais evidente, pois tivemos contato posterior, especialmente com os estudantes de graduação que participaram da realização das atividades. Muitos estudantes buscaram a partir da SIC se inserir em grupos de pesquisa da universidade, o que demonstra que as ações despertaram a curiosidade científica e os estimularam a buscar mais e novos conhecimentos. Em relação à educação básica o impacto percebido também foi grande. Houve interesse dos professores e das escolas e, a partir da SIC organizamos uma ação permanente de divulgação da neurociência em escolas públicas de Uruguaiana/RS. Esta ação está em andamento desde o segundo semestre de 2013 e busca levar a neurociência até a escola, envolvendo atividades com alunos e professores.

\section{Considerações Finais}

As atividades propostas para divulgação da neurociência durante a Semana Internacional do Cérebro 2013 em Uruguaiana/RS permitiram uma aproximação da comunidade com a universidade, com o discurso e práticas científicas, permitindo a erradicação de mitos e o combate da visão da ciência como algo além do conhecimento das pessoas comuns, permitindo outros olhares para neurociência. Adicionalmente, as ações aqui relatadas contribuíram para o amadurecimento pessoal e profissional dos organizadores como estudantes e futuros pesquisadores/profissionais da ciência do cérebro.

\section{Referências}

[1] Trópia G. Reflexões sobre o discurso na divulgação neurocientífica. Ciência \& Ensino, 2008. v. 2, n. 2.

[2] Germano, M. G. Popularização da ciência como ação cultural libertadora. In: Anais do V Colóquio Internacional Paulo Freire: Desafios à Sociedade Multicultural.; Recife, 2005. p. 1-18.

[3] Candotti E, Barros H, Germano, M. Mesa Redonda: Os desafios da Popularização da Ciência. Reunião Regional da SBPC, 2003. UFCG.

[4] Candotti E. Ciência na Educação Popular. In: Massarani L, Moreira IC, Brito F. (orgs.) Ciência e Público: caminhos da divulgação científica no Brasil. Casa da Ciência, 2002. UFRJ.

[5] Marandino, M. A pesquisa educacional e a produção de saberes nos museus de 
Imagem Pública E Divulgação Científica Da Bioquímica: Ações para divulgação da Neurociência: um relato de experiências vivenciadas no sul do Brasil

ciência. História, Ciências, Saúde - Manguinhos 2005; v. 12, 161-81.

[6] Guimarães, M.; Vasconcellos, M. das M. N. Relações entre educação ambiental e educação em ciências na complementaridade dos espaços formais e não formais de educação. Educar, 2006. n. 27, p. 147-162.

[7] Cunha, M. B. da. A percepção da ciência e tecnologia dos estudantes de ensino médio e a divulgação científica. Tese Doutorado [Educação] - Faculdade de Educação da Universidade de São Paulo, 2009.

[8] Zamboni, L. M. S. Cientistas, jornalistas e a Divulgação Científica: subjetividade e heterogeneidade no discurso da Divulgação Científica. Ed. Autores Associados, Campinas, SP. $1^{\text {a }}$ ed., 2001.

[9] Sarmento AC, Ferreira C, Oliveira I, Porto CM. Divulgação científica para o público infantil: análise da revista ciência hoje das crianças - impressa. Diálogos \& Ciência Revista da Rede de Ensino FTC, 2010. Ano IV, n. 12.

[10] Carvalho, AMP de. Ensino De Ciências: Unindo a Pesquisa e a Prática. Pioneira Thomson Learning, 2006. p. 1-17

[11] Nascimento Júnior AF, Souza, DC de. O Fazer artístico na popularização do conhecimento biológico: relato de experiências I. REMEA [periódicos na internet]. 2009. acesso em 16 de junho de 2014. Disponível em: http://www.seer.furg.br/remea/article/viewFile/3956/2352

[12] Teixeira MRF. O site Contando Ciência na Web: um instrumento de inclusão social. Inc. Soc. 2011. v. 4 n. 2, p. 19-24.

[13] Adestro S, Garbin MC, Li Min Li. ABCérebro TV program presents: science popularization on epilepsy. J Epilepsy Clin Neurophysiol 2013. v. 4, n 19, p. 74-78.

\section{Agradecimentos}

Os autores agradecem à Pró-reitoria de Extensão da Universidade Federal do Pampa, pelos recursos concedidos através de editais de fomento à extensão (PROFEXT/Unipampa) e bolsas de iniciação à extensão (PBDA/Unipampa) no ano de 2013, bem como à Sociedade Brasileira de Fisiologia (SBFis), pelos recursos concedidos através do Programa de Auxilio a Eventos 2013, à Coordenação de Aperfeiçoamento de Pessoal do Ensino Superior (CAPES), pelos recursos concedidos através do Edital Novos Talentos/CAPES, e ao SESC/Uruguaiana pelo empréstimo dos espaços. Os autores também agradecem a receptividade da direção, professores e alunos das escolas estaduais Hermeto José Pinto Bermudez, Romaguera Correa, Dr. Roberval Behegaray Azevedo e Cândido Rondon, que participaram das ações. 